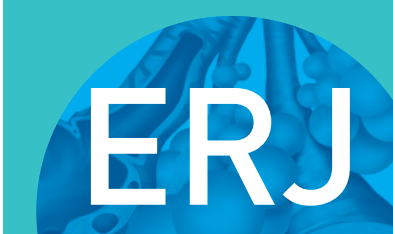

open research
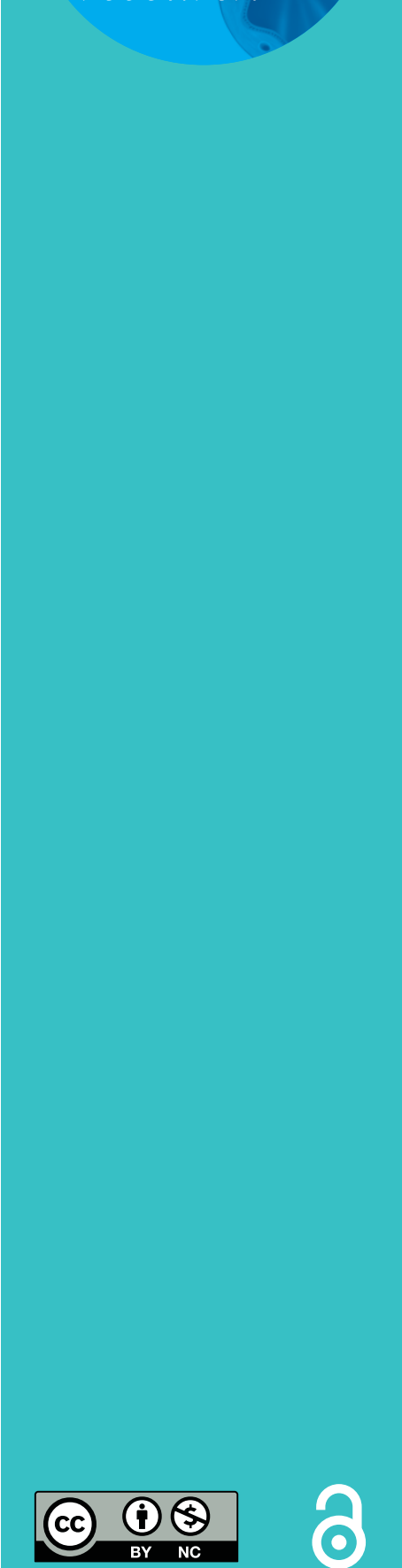

\section{Identification and treatment of T2-low asthma in the era of biologics}

\author{
Chris Kyriakopoulos (D), Athena Gogali, Konstantinos Bartziokas (i) and \\ Konstantinos Kostikas
}

Affiliation: Respiratory Medicine Dept, University of Ioannina School of Medicine, Ioannina, Greece.

Correspondence: Konstantinos Kostikas, Respiratory Medicine Dept, University of loannina School of Medicine, 45110 loannina, Greece. E-mail: ktkostikasdgmail.com

ABSTRACT Currently, and based on the development of relevant biologic therapies, T2-high is the most well-defined endotype of asthma. Although much progress has been made in elucidating T2-high inflammation pathways, no specific clinically applicable biomarkers for T2-low asthma have been identified. The therapeutic approach of T2-low asthma is a problem urgently needing resolution, firstly because these patients have poor response to steroids, and secondly because they are not candidates for the newer targeted biologic agents. Thus, there is an unmet need for the identification of biomarkers that can help the diagnosis and endotyping of T2-low asthma.

Ongoing investigation is focusing on neutrophilic airway inflammation mediators as therapeutic targets, including interleukin (IL)-8, IL-17, IL-1, IL-6, IL-23 and tumour necrosis factor- $\alpha$; molecules that target restoration of corticosteroid sensitivity, mainly mitogen-activated protein kinase inhibitors, tyrosine kinase inhibitors and phosphatidylinositol 3-kinase inhibitors; phosphodiesterase (PDE) 3 inhibitors that act as bronchodilators and PDE4 inhibitors that have an anti-inflammatory effect; and airway smooth muscle mass attenuation therapies, mainly for patients with paucigranulocytic inflammation.

This article aims to review the evidence for noneosinophilic inflammation being a target for therapy in asthma; discuss current and potential future therapeutic approaches, such as novel molecules and biologic agents; and assess clinical trials of licensed drugs in the treatment of T2-low asthma.

@ERSpublications

Th2-low endotype of asthma occurs in $50 \%$ of asthma patients. Novel targeted treatment with biologic agents is not currently approved. Nevertheless, other therapeutic options exist. Licensed drugs, novel molecules and biologics agents are under development. https://bit.ly/3bW7G0Q

Cite this article as: Kyriakopoulos $\mathrm{C}$, Gogali A, Bartziokas $\mathrm{K}$, et al. Identification and treatment of T2-low asthma in the era of biologics. ERJ Open Res 2021; 7: 00309-2020 [https://doi.org/10.1183/ 23120541.00309-2020].

Received: 25 May 2020 | Accepted after revision: 8 Sept 2020

Copyright OERS 2021. This version is distributed under the terms of the Creative Commons Attribution NonCommercial Licence 4.0. For commercial reproduction rights and permissions contact permissions@ersnet.org 


\section{Background}

Asthma is a chronic inflammatory disorder of the airways, characterised by reversible expiratory flow limitation and bronchial hyperresponsiveness (an increased sensitivity of the airways resulting in bronchoconstriction) to a variety of triggers. It presents with symptoms such as wheezing, shortness of breath, cough and chest tightness that vary over time and in severity [1]. This is a rough description, as asthma is not a homogenous disease in terms of disease natural history, severity and response to treatment. It has variable clinical presentations (phenotypes) and distinct underlying pathophysiological pathways (endotypes) responsible for this heterogeneity [2]. Based on the presence of T-helper cell type 2 (Th2)-driven inflammatory responses (interleukin (IL)-4-, IL-5- and IL-13-mediated), two major asthma endotypes, Th2 and non-Th2, have been described. The recent discovery of type- 2 innate lymphoid cells and the fact that they can release Th-2 cytokines (predominantly IL-5 and IL-13), contributing to the type 2 (T2)-high signature, led to the better categorisation of asthma into T2-high or T2-low or non-T2. Currently, based on the existence of T2 biomarkers and the development of relevant biologic therapies, T2-high asthma is the most well-defined endotype [3]. T2-low asthma presents with either neutrophilic or paucigranulocytic inflammation, tends to be more resistant to inhaled corticosteroids, and it involves various asthma phenotypes, related to obesity, smoking, late onset (usually after the age of 40 years) or occupational exposures [2]. Thus, there is an unmet need for the identification of biomarkers that can help the diagnosis and the endotyping of T2-low asthma. The therapeutic approach of T2-low asthma is a problem urgently needing resolution for two main reasons: first, these patients have poor response to steroids, the cornerstone of asthma treatment [4], and second, they are not candidates for the newer targeted treatment with biologic agents such as anti-IgE or anti-IL-5 [5]. This review aims to discuss older as well as more recent therapeutic approaches, such as novel small molecules and biologic agents, in the treatment of T2-low asthma.

\section{Definition of T2-low asthma}

At present, a solid definition of T2-low asthma has not been established. Although some emphasis has been placed on noninvasive biomarkers for the detection of T2-high asthma (i.e. T2 cytokines such as IL-4, IL-5 and IL-13, exhaled nitric oxide fraction $\left(F_{\text {eNO }}\right)$, serum periostin, total IgE, blood and sputum eosinophils), the exact cut-off points for these markers are debatable [6]. Furthermore, these T2 markers may have low concordance when measured in the same patient $[7,8]$ and may be subject to significant variability over time and in response to asthma treatments [9]. The most important characteristic of these biomarkers is that they are characterised by high specificity, but low sensitivity, rendering them more useful in the identification of T2-high patients. In clinical practice, the most useful tool to identify T2-low asthma phenotype is the absence of any evidence of increased values in the aforementioned biomarkers of T2-high asthma. Additionally, it seems that there is an association of T2-low asthma with obesity, smoking, pollutants, viral or bacterial infections and advanced age. In terms of pathophysiology, T2-low asthma is usually characterised by neutrophilic (NA) or the paucigranulocytic (PGA; absence of sputum eosinophilia or neutrophilia) phenotype of inflammation [10] and Th1 and/or Th17 cells seem to be the key effector cells in this setting.

Several studies have related asthma severity to airway inflammation. DuONG-QuY [11] has related eosinophilic inflammation to asthma management, asthma control and to predicting response to inhaled corticosteroids (ICS), through $F_{\mathrm{eNO}}$ measurement. KuO et al. [12] have shown that type 2 inflammation, as reflected by $F_{\mathrm{eNO}}$, serum immunoglobulin (Ig)E and blood eosinophils, plays a central role in small airways dysfunction in adults with moderate to severe persistent asthma. Lous et al. [13] demonstrated the connection between clinical activity, bronchial hyperresponsiveness and lung function to eosinophilic, and to a lesser degree, neutrophilic inflammation. In a recent study Scioscia et al. [14] showed a positive correlation between $17 \beta$-estradiol concentrations and neutrophil levels in in the sputum of female patients with postmenopausal severe asthma, and their results confirmed the central role of neutrophils in the pathogenesis of some forms of severe late-onset asthma. It is possible that increased airway remodelling is an important future risk factor for asthma, inducing further decline in pulmonary function, increased BHR, and exacerbation rate [15].

\section{Neutrophilic phenotype}

The percentage of neutrophils in the sputum of healthy controls ranges from $0 \%$ to $64 \%[16,17]$, the aetiology of which may be shear stress in the mucosa during the induction process, the influence of specific resident microbiota or prior exposures to environmental agents. Airway neutrophilia may be associated with pollution [18], smoking [19], workplace agents [20], acute infections [21], chronic airway infections (e.g. in bronchiectasis) [22] or with an altered airway microbiome [23]. NA is common in smokers with asthma [19], obese asthmatics [24] and occupational asthma [25, 26]. The NA phenotype is characterised by a neutrophilic proportion of $\geqslant 64 \%$ in induced sputum $[27,28]$, although different cut-off values have been used in different studies ranging from $40 \%$ to $76 \%$ [29]. It is uncertain which cut-off 
value correlates with activated neutrophils promoting the pathogenic process [30]; nevertheless, sputum neutrophil counts have been associated with disease severity [31]. It is prudent to mention that sputum total cell count $>15 \times 10^{6}$ cells.g ${ }^{-1}$ with a neutrophil percentage $>64 \%$ is a strong indication of lower airway tract infection [32].

The NA phenotype accounts for 5-22\% of asthmatic patients [33], often related to more severe disease with worse pulmonary function and poor asthma control [13]. Patients with NA are less responsive to ICS [34]; however, it is unclear if this corticosteroid insensitivity is related to the presence of neutrophils or if it is induced by the mechanisms underlying neutrophil recruitment [35].

Although blood eosinophils correlate with sputum eosinophilia, this is not applicable in the NA phenotype, in which blood neutrophilia cannot predict airway neutrophilia [30]. However, there are several candidate biomarkers to define NA, apart from the differential sputum cell counts; they are not applicable in clinical daily practice. Several cytokines are associated with neutrophilic airway inflammation. IL-6 is a pleiotropic cytokine produced by various cell types in response to a wide range of inflammatory stimuli. It is considered an indicator of metabolic dysfunction as well as asthma severity and has been identified as a potential candidate biomarker in a study with obese asthmatic patients [36]. Increased levels of IL-8, which is known to activate neutrophils, have been observed in patients with NA [37] and were linked to sputum neutrophil counts [38]. Other important biomarkers of neutrophil induction are myeloperoxidase and neutrophil elastase which can be discovered in induced sputum samples [39]. Tumour necrosis factor (TNF)- $\alpha$ [40], IL-23 [41] and IL-17 [42] are also related to neutrophilic inflammation in asthma. MAES et al. [43] have suggested that certain micro-RNAs in sputum might be able to accurately identify neutrophilic airway inflammation, but most clinicians are unable to obtain satisfactory sputum samples from these patients. Transcriptomic analysis of airway samples in severe neutrophilic asthma found a strong upregulation of mucins, IL-17-inducible chemokines (CXCL1, CXCL2, CXCL3 and CSF-3) and the neutrophil chemoattractants CXCL8 (IL-8), CCL3 and Galectin-3 (LGALS3) [44]. NA is associated with airway colonisation by bacteria including Haemophilus influenzae [45] and Moraxella catarrhalis [46], which might induce Th17 responses [47]. In addition, neutrophilic asthma is associated with upregulation of oxidative stress responses and matrix metalloproteases [48], including matrix metalloprotease (MMP)-9, a type IV collagenase involved with CXCL8-induced neutrophilia [49]. MMPs are increased in airway samples from asthmatic smokers and in severe neutrophilic asthma and are implicated in tissue remodelling [50].

\section{Paucigranulocytic phenotype}

Asthmatic patients with both neutrophil levels $<64 \%$ and eosinophil levels $<2 \%$ are classified as having PGA [29]. The prevalence of this phenotype in asthmatic patients ranges from $17 \%$ to $48 \%$ in different studies [30,37]. Although little is known about the PGA phenotype, in the majority of patients with PGA the absence of inflammatory cells is usually related to appropriate anti-inflammatory treatment that achieves adequate asthma control [30]. Even though PGA is related to better lung function, a substantial proportion of patients with PGA (21.7\%) are characterised as having severe refractory asthma, and $14.8 \%$ of patients with PGA have an Asthma Control Test score $<19$, suggesting that only this subpopulation of PGA is not well controlled despite the absence of inflammatory cells in their sputum [38]. Surprisingly, patients with PGA with severe refractory asthma presented with higher levels of $F_{\text {eNO }}$ and sputum IL-13 and IL-8 compared with those with mild-to-moderate asthma [37, 41]. Animal models demonstrate that processes stimulating airway hyperresponsiveness and airway smooth muscle thickening occur

TABLE 1 Differential diagnostic characteristics of type 2 (T2)-low versus T2-high asthma

\begin{tabular}{lcc} 
& T2-low asthma & T2-high asthma \\
\hline Onset & Late & Early \\
Symptoms & Mostly significant & May be significant \\
Obesity & Often present & May be present \\
Smoking & Often present & May be present \\
Response to inhaled corticosteroids & Often poor & Usually good \\
Severity of asthma & Often difficult to treat & Mild to severe \\
Asthma control & Often poor & Variable \\
Sputum eosinophils & Absent & Normal or high levels \\
Sputum neutrophils & Frequently present & May be present \\
Exhaled nitric oxide & Usually normal & Elevated or normal \\
Airways & Often present & May be present
\end{tabular}


independently from inflammation and are a consequence of a loss of negative homeostatic processes, advancing eventually to the pathogenesis of asthma in PGA phenotype [51].

The main clinical and pathophysiological characteristics that can help distinguish T2-low from T2-high asthma are shown in table 1, while possible pathogenetic pathways, current drug targets and those in the development pipeline are shown in figure 1.

\section{Current therapeutic options}

Presently, there are no specific therapeutic interventions for patients with PGA. A crucial and low-cost, but often overlooked, intervention that may dramatically improve asthma control in patients with NA is smoking cessation and avoidance of exposure to environmental/occupational pollutant agents. In smokers with NA, smoking cessation and avoidance of second-hand smoke resulted in the reduction of neutrophilic inflammation and improved asthma control and lung function [52].
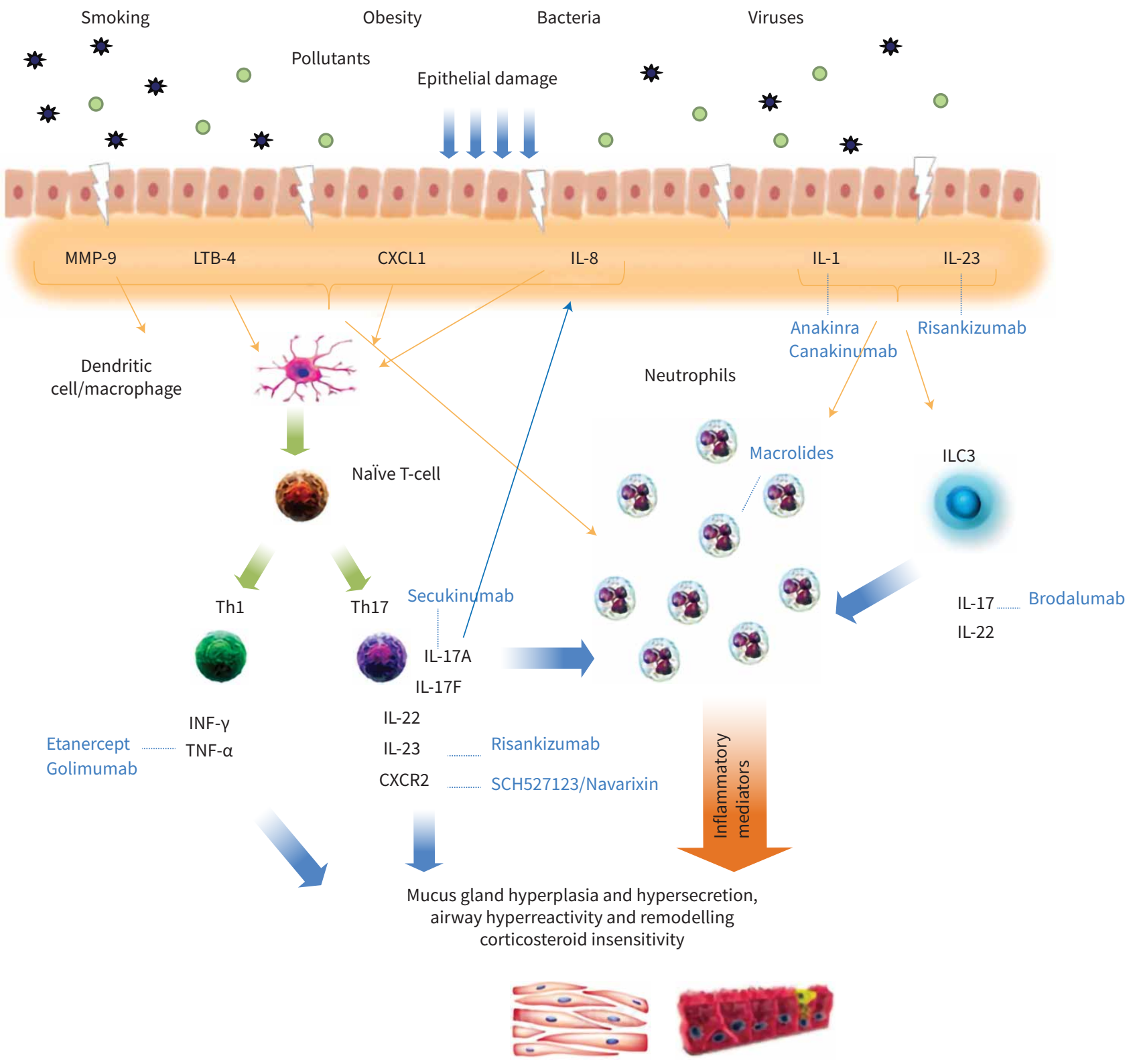

Bronchial thermoplasty

FIGURE 1 Type 2-low asthma pathways and potential therapeutic targets. MMP: matrix metalloprotease; LTB: leukotriene B; IL: interleukin; Th: T-helper cell; INF: interferon; TNF: tumour necrosis factor; ILC: innate lymphoid cells. 
Other treatment approaches currently available for T2-low asthma address noninflammatory pathways possibly involved in its pathogenesis. Tiotropium has been recently included as a new add-on treatment for Global Initiative for Asthma steps 4 and 5 in patients aged $\geqslant 18$ years with a history of exacerbations. Its clinical efficacy has been demonstrated in patients with fixed airway obstruction, an asthma phenotype usually associated with neutrophilic airway inflammation [53] and in patients with low sputum eosinophils (and possibly T2-low asthma) [54].

Macrolides are often administered in refractory NA, although these antibiotics promote an enhanced risk of adverse events and promote bacterial resistance [55]. Macrolides exhibit their beneficial effects in NA with three mechanisms: 1) they exert antibacterial [56] and antiviral actions [57]; 2) they demonstrate immunomodulatory effects and anti-inflammatory activity through inhibition of transcription factors such as $\mathrm{NF \kappa B}$, reduction of activation and migration of neutrophils [58], revitalisation of corticosteroid sensitivity by inhibiting the phosphatidylinositol 3-kinase (PI3K) pathway and restoring histone deacetylase-2 [59] and by attenuating TNF- $\alpha$ and IL-17 immune responses [58]; and 3) they display prokinetic properties, thus diminishing the pernicious consequences of reflux and aspiration events [60]. Although not explicitly highlighted, macrolides reduce the sequence of reflux effects that include micro and macro aspiration of gastric contents, bronchoconstriction mediated by vagal reflexes [61] and neurogenic inflammation in the lung [62]. Erythromycin has been shown to promote gastric and small-bowel motility [63], increase lower oesophagal sphincter pressure [64] and affect gallbladder function [65]. Additionally, azithromycin exhibits action as a motilin receptor agonist [66] and enhances gastric motility in healthy subjects [67]. In patients with impaired gastrointestinal motility, azithromycin has shown comparable positive effects on gastric and duodenal motility to erythromycin [66]. In one study which included smoking asthmatics, the use of azithromycin did not induce amelioration of asthma control or lung function [68]. In other reports, azithromycin significantly improved asthma exacerbations and quality of life in both eosinophilic and noneosinophilic asthma [69, 70]. Moreover, the use of clarithromycin in patients with NA resulted in the reduction of neutrophilic inflammation followed by improvement in asthma control [71]. In a large randomised, double-blind study in moderate-to-severe asthma in Australia (AMAZES), azithromycin reduced exacerbations and improved Asthma Quality of Life Questionnaire scores [70]. Moreover, current European Respiratory Society/American Thoracic Society severe asthma guidelines recommend the use of azithromycin in severe asthma that does not respond to treatment [72].

Bronchial thermoplasty could be a possible therapeutic approach, but its long-term effectiveness or adverse events have not been fully assessed in PGA [73]. Bronchial thermoplasty administers targeted thermal energy to the airway wall, ablating airway smooth muscle (ASM) cells with a subsequent decrease in the ASM mass [74], while it has been verified that thermoplasty also regulates mucosal inflammatory responses and collagen deposition [75]. This intervention has been observed to reduce asthma exacerbations and to improve lung function and health-related quality of life. Moreover, macrolides may be an effective option in PGA, since the potential effects of azithromycin have been observed irrespective of the underlying airway inflammation [69].

The use of statins in clinical trials demonstrated alterations in several inflammatory markers, but failed to show improvement in asthma control or lung function [76].

Given the high prevalence of obesity in patients with T2-low asthma, weight reduction along with exercise and dietary modification should be included in the therapeutic plan of those patients. Several clinical studies have shown that weight loss in obese patients not only improves asthma symptoms, but also contributes to a reduction in doses of rescue medication. It also reduces the risk for severe asthma exacerbations and improves asthma-related quality of life, perhaps through improvements in lung function [77-79].

\section{Future therapeutic targets}

Although no specific treatments have been made available so far, according to the pathophysiology of T2-low asthma, some potential targets for therapeutic interventions are summarised herein [80]. An overview of the major biologic approaches currently in development for T2-low asthma management is shown in table 2.

\section{CXCR2/IL-8}

IL-8 is a potent chemoattractant that regulates activation and migration of neutrophils to the site of inflammation through the high-affinity CXCR2 receptor. Increased levels of IL-8 in sputum often precede asthma exacerbations in severe asthmatics and coincide with the development of late-phase airflow obstruction in patients with atopy [97]. IL-8 is also elevated in the serum of those patients, but its role as a biomarker demonstrating disease activity remains disputable. CXCR2 is a common chemokine receptor, 
TABLE 2 Overview of the major biologic approaches currently in development for type 2 low asthma management

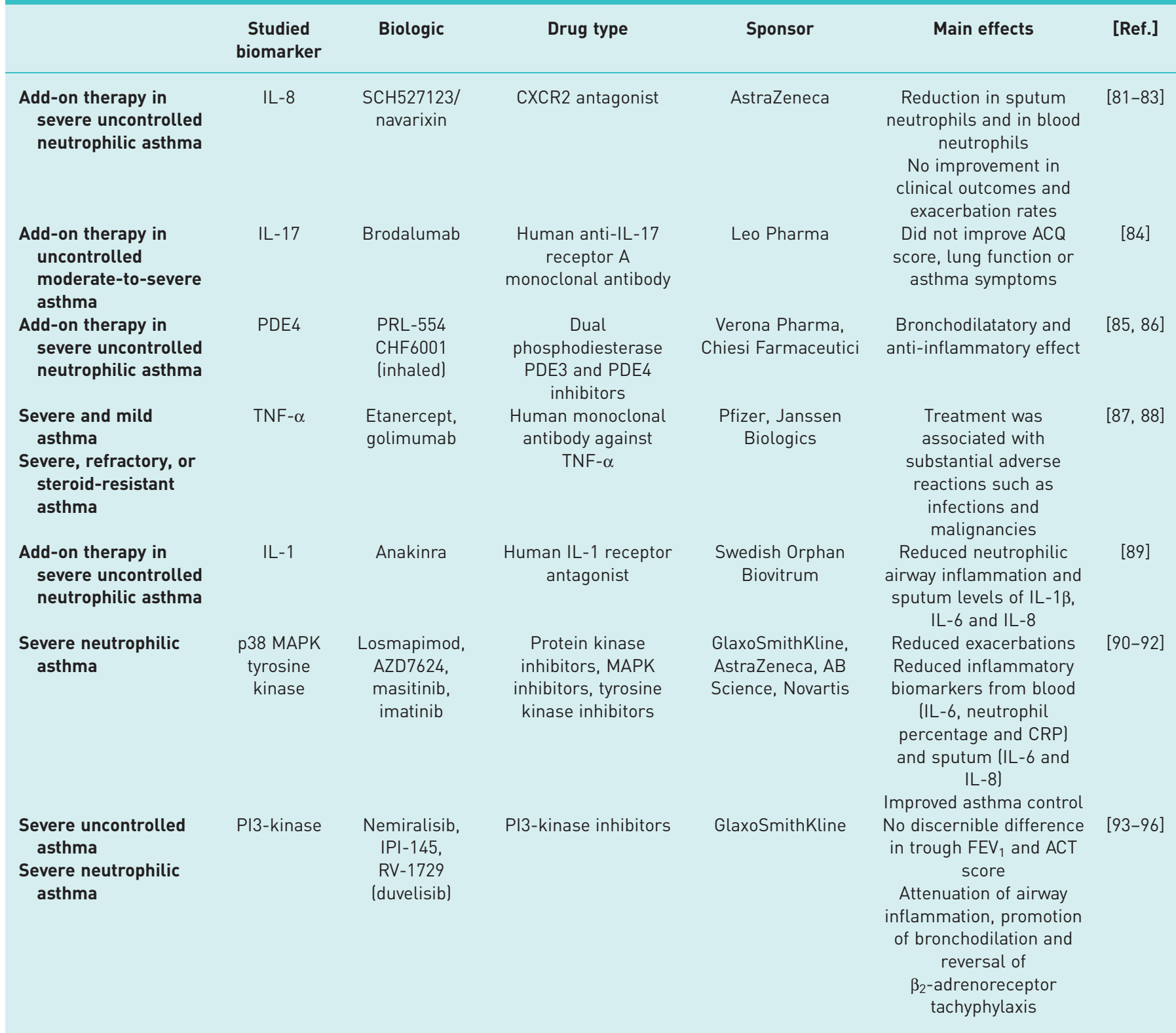

IL: interleukin; ACQ: Asthma Control Questionnaire; PDE: phosophdiesterase; TNF: tumour necrosis factor; MAPK: mitogen-activated protein kinase; CRP: C-reactive protein; PI3: phosphoinositide 3; FEV ${ }_{1}$ : forced expiratory volume in $1 \mathrm{~s} ; \mathrm{ACT}$ : Asthma Control Test.

which is expressed on the surface of neutrophils. The tripeptide PGP, which is generated from extracellular matrix proteins through the action of matrix metalloproteinases, is also a neutrophil chemoattractant (matrikine) that activates CXCR2 [98]. These chemokines also attract circulating monocytes, which differentiate within the lung to macrophages that are thought to guide neutrophilic inflammation [99]. This implicates that blocking CXCR2 could be a tempting therapeutic approach to the treatment of neutrophilic inflammation since small molecule inhibitors to these G-protein receptors have now been developed.

In a phase 2 study of patients with moderate-to-severe asthma with high neutrophils count at baseline (>40\%), CXCR2 antagonist SCH527123/navarixin demonstrated a significant reduction in sputum neutrophils by $36.3 \%$ compared to a $6.7 \%$ increase in the placebo group ( $p=0.03)$, but no significant improvement of asthma control was established and no change to forced expiratory volume in $1 \mathrm{~s}\left(\mathrm{FEV}_{1}\right)$ was proved [81, 82]. In a subsequent randomised clinical trial, using a CXCR2 antagonist (AZD5069) as add on therapy for patients with severe asthma $45 \mathrm{mg}$ twice daily $(\mathrm{n}=161)$ for up 12 months resulted in a 
sustained $\sim 25 \%$ reduction in blood neutrophils, reversible on discontinuation of treatment. In severe asthmatics, 5, 15 or $45 \mathrm{mg}$ twice daily for 6 months neither reduced the rate of exacerbations nor improved $\mathrm{FEV}_{1}$ or total asthma symptom score. A statistically significant reduction of sputum neutrophils by $69 \%$ was found in bronchiectasis patients after $80 \mathrm{mg}$ twice daily for 4 weeks. There was no consistent increase in the rate of infections [82]. Another phase 1 clinical trial treatment of AZD8309, a CXCR2 antagonist, demonstrated a mean $77 \%$ reduction in total sputum cells $(\mathrm{p}<0.001)$ and $79 \%$ reduction in sputum neutrophils $(\mathrm{p}<0.05)$ compared with placebo after lipopolysaccharide (LPS) challenge. In addition, there was a reduction in neutrophil elastase activity $(\mathrm{p}<0.05)$ and CXCL1 $(\mathrm{p}<0.05)$ and trends for a reduction in sputum macrophages (47\%), leukotriene $\mathrm{B}_{4}(39 \%)$ and CXCL8 (52\%). The clinical trial was discontinued for asthma [83]. Overall, although CXCR2 antagonists seemed to be promising molecules as an add-on therapy for severe uncontrolled NA, they were not able to improve clinical outcomes and exacerbation rates $[81-83]$.

\section{IL-17}

IL-17A and IL-17F are produced by Th17 cells and trigger the production of IL- 8 and CXCL1 from epithelial cells. Moreover, they are neutrophil chemoattractants [100], while Th1 cells are connected to neutrophilic inflammation, by producing interferon- $\gamma$ and TNF- $\alpha$. Neutrophilic inflammation generated by this pathway leads to airway injury and remodelling, hyperplasia and hypersecretion of the mucus gland and corticosteroid insensitivity [76]. It has been recorded that severe NA is related to higher levels of Th17 cytokines, primarily CXCL1, CXCL10, CCl-2, IL-6 and IL-8 in comparison to other inflammatory endotypes [101]. In patients with asthma, elevated levels of IL-17A and IL-17F are found in bronchoalveolar lavage (BAL) and airway tissue biopsies and positively correlate with the level of disease severity and neutrophil inflammation [102]. Furthermore, IL-17A acts on airway smooth muscle cells and mediates airway hyperresponsiveness. IL-17A and IL17F bind to receptor complexes, with IL-17RA serving as the common subunit. Nevertheless, a recent study with mice models suggested that IL-17F, rather than IL-17A, underlies airway inflammation in steroid-insensitive asthma [103].

Secukinumab, an IL-17A blocker, administration in healthy volunteers who evolved acute neutrophilic airway inflammation following an ozone challenge did not demonstrate a significant change in the total number of sputum neutrophils from baseline. In a subsequent phase 2 study of AIN457 (secukinumab) in patients with uncontrolled asthma, there was no difference in Asthma Control Questionnaire (ACQ) score leading to its termination [104]. Brodalumab is a human monoclonal antibody that blocks the activity of IL-17A, IL-17B and IL-25 through binding to IL-17RA. In a randomised controlled trial recruiting patients with uncontrolled moderate-to-severe asthma on inhaled corticosteroids, brodalumab failed to show a change in ACQ score, asthma symptoms or lung function in the overall study population. However, it did show a trend towards improvement in ACQ score in a small subgroup of patients with high bronchodilator reversibility [84]. However, this drug led to significant mental health issues in clinical trials, thus resulting in discontinuation of further development for asthma. Another formulation of secukinumab (CJM112), an anti-IL17A antibody, is now in phase 2 randomised clinical trial for patients with inadequately controlled moderate-to-severe asthma with low IgE and low circulating eosinophil levels, but no results have been announced yet [105].

\section{Phosphodiesterases}

Most phosphodiesterase (PDE) isoenzymes are expressed in lung tissue, including PDE1, PDE2, PDE3, PDE4, PDE5, PDE7, PDE8 and PDE9; however, PDE3 and PDE4 have been the primary targets for drug therapy. The PDE4 isoenzyme is found in most inflammatory and immune cells, including T-cells, eosinophils, neutrophils, B-cells, monocytes, macrophages and dendritic cells. In several pre-clinical studies, PDE4 inhibitors have been shown to suppress bronchial hyperresponsiveness, eosinophil infiltration and production of histamine, leukotrienes and cytokines; however, they have significant side-effects when administered orally. PDE3 inhibitors act as bronchodilators, while PDE4 inhibitors have an anti-inflammatory effect [106]. PRL-554, an inhaled PDE3/4 inhibitor is now in a phase 1 trial for severe asthma. It is an analogue of trequinsin and, like trequinsin, is a highly selective inhibitor of the phosphodiesterase enzyme, PDE3. Indeed, it is $>3000$ times more potent against PDE3 than against PDE4. Ensifentrine (RPL554), a nebulised PDE3/4 inhibitor, demonstrated a significant dose-dependent bronchodilator response in asthma patients [76, 85, 86]. CHF6001, an inhaled PDE4 inhibitor, was evaluated in a phase 2 trial; the drug was safe and well tolerated at daily doses up to $4800 \mu \mathrm{g}$ for 14 days in healthy volunteers. No results have been announced yet about drug efficacy [107].

TNF- $\alpha$

TNF- $\alpha$ is secreted by lymphocytes, mast cells and macrophages and promotes bronchial hyperresponsiveness and sputum neutrophilia, thus was considered as an attractive target for severe 
asthma [87]. Etanercept (anti-TNF- $\alpha$ ) showed short-term and modest clinical efficacy for severe and mild asthma, respectively [88]. Mild reduction in the number of exacerbations and improvement in wheezing was observed in patients with moderate uncontrolled asthma, who were under monotherapy with ICS, after administration of infliximab or adalimumab [108]. Golimumab lowered risk of asthma exacerbations; nevertheless, its trial on severe persistent asthma ended at phase 2 due to incidences of malignancies and infections such as pneumonia [87]. Given the possible beneficial role of TNF- $\alpha$ in severe NA, future studies may need to evaluate the long-term risk-benefit profile of these agents in such patients.

\section{IL-1}

IL-1 $\beta$ and IL-1 receptor antagonist genes have both been associated with asthma risk [109]. In humans, IL-1 $\beta$ is increased in BAL fluid and sputum of patients with asthma compared to normal volunteers [110], with airway macrophages from asthma patients also having increased expression of IL-1 $\beta$ [111]. In addition, IL-1 $\beta$ is increased in BAL fluid from persons with symptomatic asthma versus those with asymptomatic asthma [112], and there is an increased expression of this cytokine in the airway epithelium of asthmatics [112]. Furthermore, IL-1 $\beta$ appears to be involved in the recruitment of airway neutrophils by LPS in rat models [113] and is upregulated in the airway after LPS inhalation challenge in humans [114]. Canakinumab, a fully human immunoglobulin IgG1k monoclonal antibody against IL-1 $\beta$, was originally developed as a potential treatment for asthma, but is no longer listed for that indication [115]. A study of anakinra, an IL-1 $\beta$ antagonist, in NA demonstrated a significant reduction of airway neutrophilia compared to placebo; subjects tolerated the treatment well, without an increased frequency of infections that have been attributable to anakinra [89].

\section{IL-6}

IL-6 in sputum is associated with a lower $\mathrm{FEV}_{1}$ in patients with mixed eosinophilic-neutrophilic bronchitis [116]. In mice, allergen exposure increased lung IL-6, and IL-6 was produced by dendritic cells and alveolar macrophages. Loss of function of IL-6 signalling abrogated elevations of eosinophil and neutrophil recruiting cytokines/chemokines and allergen-induced airway inflammation in mice. The association of pleiotropic cellular airway inflammation with IL-6 using human and animal data suggests that exacerbations of asthma, particularly those with a combined eosinophilic and neutrophilic bronchitis, may respond to therapies targeting the IL-6 pathway [117]. Sirukumab, an IL-6 antagonist for patients having uncontrolled severe asthma despite the use of high-dose ICS in combination with long-acting $\beta$-agonist (LABA) is now in a phase 2a trial [118].

\section{IL-22 and IL-23}

Currently, one phase 2 ongoing clinical trial for anti-IL-23 monoclonal antibody (risankizumab) is attempting to investigate the efficacy and safety profile of this agent in T2-low severe asthmatic patients (clinicaltrials.gov identifier NCT02443298). There are no ongoing studies on IL-22 targeted therapy in asthma.

\section{Protein kinases}

Several p38 mitogen-activated protein kinases (MAPK) inhibitors have shown to restore corticosteroid sensitivity in peripheral blood mononuclear cells from patients with severe asthma [119]. A phase 2 study of PF03715455 was conducted and was terminated due to business reasons, without any safety or efficacy concerns [120]. A post hoc analysis of a 6-month clinical trial with losmapimod (GW856553) in COPD, but not in asthma, reported a reduction in exacerbations in a subgroup of patients with a blood eosinophil count $\leqslant 2 \%$, which may suggest a preferentially beneficial effect of p38 MAPK inhibitors in noneosinophilic inflammation [90]. In COPD, AZD7624, an inhaled p38 $\alpha$ and p38 $\beta$ inhibitor, significantly reduced additional inflammatory biomarkers from sputum (IL-6, IL-8 and macrophage inflammatory protein (MIP)-1 $\beta$ ) and blood (neutrophil percentage, IL-6, MIP-1 $\beta$ and C-reactive protein), but demonstrated no benefit concerning the reduction of exacerbations [91].

Inhibition of the stem cell factor/c-kit receptor pathway leads to a significant decrease of the mast cell population, histamine levels, IL-4 production and airway hyperresponsiveness. Growth factors such as platelet-derived growth factor (PDGF) contribute to bronchial remodelling, a characteristic of severe asthma [121]. Masitinib and imatinib are tyrosine kinase inhibitors, targeting stem cell factor receptor/ c-kit and PDGF receptor. A phase 2a study of masitinib in severe, uncontrolled, corticosteroid-dependent asthmatics was conducted, showing no significant difference to the corticosteroid weaning process; in contrast, a significant improvement in asthma control was observed [92].

PI3K and Janus-activated kinases (JAK) modulate lymphocyte activation. JAK and PI3K inhibitors reduced the cytokine level through direct effects on T-cell activation in both asthma and healthy BAL cells. Nemiralisib (GSK2269557), a once-daily inhaled PI3K $\delta$ inhibitor underwent a phase 2 clinical trial for 
patients with severe, uncontrolled asthma. It exhibited a significant reduction in sputum IL-5, IL-13, IL-6 and IL-8 level; however, it had no discernible difference in trough FEV 1 and Asthma Control Test score [93]. PI3K $\delta$ and $\gamma$ isoforms are involved in inflammatory cell recruitment and activation and dual PI3K $\delta / \gamma$ inhibitors, such as TG100-115 and IPI145 reduces airway inflammation induced by allergen or cigarette smoke in murine models $[94,95]$ and restored corticosteroid sensitivity in the smoke model [95]. A phase 2 study of RV1729, an inhaled PI3K $\gamma / \delta$ inhibitor, demonstrated attenuation of airway inflammation, promotion of bronchodilation and reversal of $\beta_{2}$-adrenoreceptor tachyphylaxis [96]. Both IPI-145 (duvelisib) and RV-1729 are PI3Kס/PI3K $\gamma$ combination inhibitors that are being developed for clinical use $[94,95]$. Furthermore, the combination of dexamethasone with either a JAK or PI3K $\delta$ inhibitor showed an additive anti-inflammatory effect [122]. Recently, an inhaled inhibitor of JAK1 and intravenous administration of human mesenchymal stem cells attenuated neutrophilic inflammation in an experimental murine of allergic asthma [123]. Overall, the inhibition of PI3K blunts mucus production, prevents mast cell degranulation, deters immune cell recruitment and facilitates bronchodilation, all of which are potentially beneficial in asthma [124], but these need to be proven in future clinical trials.

\section{Conclusions}

Treating T2-low asthma is a therapeutic challenge, mainly because its mechanisms are not fully understood. Although much progress has been made in elucidating T2-high inflammation pathways, no specific biomarkers for T2-low asthma have been identified. Neutrophilic airway inflammation mediator targets, including IL-8, IL-17, IL-1, IL-6, IL-23, TNF- $\alpha$ or airway smooth muscle mass attenuation therapies (for paucigranulocytic inflammation) are under investigation. Failed previous attempts with some of the agents targeting these mechanisms may have been due to the poor characterisation of severe T2-low asthma. Further studies are necessary to accurately characterise T2-low pathogenetic processes to assess novel evidence-based treatments.

Conflict of interest: C. Kyriakopoulos has nothing to disclose. A. Gogali has nothing to disclose. K. Bartziokas has nothing to disclose. K. Kostikas was an employee and shareholder of Novartis Pharma AG until 31 October 2018; he has received honoraria for presentations and consultancy fees from AstraZeneca, Boehringer Ingelheim, Chiesi, CSL Behring, ELPEN, GSK, Menarini, Novartis and Sanofi; his department has received funding and grants from AstraZeneca, Boehringer Ingelheim, Chiesi, ELPEN, GSK, Innovis, Menarini, Novartis and NuvoAir.

\section{References}

1 Global Initiative for Asthma (GINA). 2019 Pocket Guide for Asthma Management: For Adults and Children Over 5 Years. Available from http://ginasthma.org/

2 Kuruvilla ME, Lee FE-H, Lee GB. Understanding asthma phenotypes, endotypes, and mechanisms of disease. Clin Rev Allergy Immunol 2019; 56: 219-233.

3 van Rijt L, von Richthofen $\mathrm{H}$, van Ree R. Type 2 innate lymphoid cells: at the cross-roads in allergic asthma. Semin Immunopathol 2016; 38: 483-496.

$4 \quad$ Gaga M, Zervas E, Samitas K, et al. Severe asthma in adults. Clin Chest Med 2012; 33: 571-583.

5 Holguin F, Cardet JC, Chung KF, et al. Management of severe asthma: a European Respiratory Society/American Thoracic Society guideline. Eur Respir J 2020; 55: 1900588.

6 Shah SP, Grunwell J, Shih J, et al. Exploring the utility of noninvasive type 2 inflammatory markers for prediction of severe asthma exacerbations in children and adolescents. J Allergy Clin Immunol Pract 2019; 7: 2624-2633.

7 Hastie AT, Moore WC, Li H, et al. Biomarker surrogates do not accurately predict sputum eosinophil and neutrophil percentages in asthmatic subjects. J Allergy Clin Immunol 2013; 132: 72-80.

8 Haughney J, Morice A, Blyth KG, et al. A retrospective cohort study in severe asthma describing commonly measured biomarkers: eosinophil count and IgE levels. Respir Med 2018; 134: 117-123.

9 Mathur SK, Fichtinger PS, Evans MD, et al. Variability of blood eosinophil count as an asthma biomarker. Ann Allergy Asthma Immunol 2016; 117: 551-553.

10 Papaioannou AI, Diamant Z, Bakakos P, et al. Towards precision medicine in severe asthma: treatment algorithms based on treatable traits. Respir Med 2018; 142: 15-22.

11 Duong-Quy S. Clinical utility of the exhaled nitric oxide (NO) measurement with portable devices in the management of allergic airway inflammation and asthma. J Asthma Allergy 2019; 12: 331-341.

12 Kuo CR, Jabbal S, Lipworth B. Is small airways dysfunction related to asthma control and type 2 inflammation? Ann Allergy Asthma Immunol 2018; 121: 631-632.

13 Louis R, Lau LCK, Bron AO, et al. The relationship between airways inflammation and asthma severity. Am J Respir Crit Care Med 2000; 161: 9-16.

14 Wardzyńska A, Pawełczyk M, Rywaniak J, et al. Circulating microRNAs and T-cell cytokine expression are associated with the characteristics of asthma exacerbation. Allergy Asthma Immunol Res 2020; 12: 125-136.

15 Scioscia G, Carpagnano GE, Lacedonia D, et al. The role of airways $17 \beta$-estradiol as a biomarker of severity in postmenopausal asthma: a pilot study. J Clin Med Res 2020; 9: 2037.

16 Wen Y, Reid DW, Zhang D, et al. Assessment of airway inflammation using sputum, BAL, and endobronchial biopsies in current and ex-smokers with established COPD. Int J Chron Obstruct Pulmon Dis 2010; 5: 327-334.

17 Pirogov AB, Prikhod'ko AG, Perelman YM, et al. Inflammatory pattern of the bronchial mucosa in patients with asthma with airway hyperresponsiveness to hypoosmotic stimulus. Bull Exp Biol Med 2016; 161: 550-553. 
Alexis NE, Carlsten C. Interplay of air pollution and asthma immunopathogenesis: a focused review of diesel exhaust and ozone. Int Immunopharmacol 2014; 23: 347-355.

Shimoda T, Obase Y, Kishikawa R, et al. Influence of cigarette smoking on airway inflammation and inhaled corticosteroid treatment in patients with asthma. Allergy Asthma Pro 2016; 37: 50-58.

Tarlo SM, Lemiere C. Occupational asthma. N Engl J Med 2014; 370: 640-649.

Yang X, Jiang Y, Wang C. Does IL-17 respond to the disordered lung microbiome and contribute to the neutrophilic phenotype in asthma? Mediators Inflamm 2016; 2016: 6470364.

Simpson JL, Grissell TV, Douwes J, et al. Innate immune activation in neutrophilic asthma and bronchiectasis Thorax 2007; 62: 211-218

Green BJ, Wiriyachaiporn S, Grainge C, et al. Potentially pathogenic airway bacteria and neutrophilic inflammation in treatment-resistant severe asthma. PLoS One 2014; 9: e100645.

Denlinger LC, Phillips BR, Ramratnam S, et al. Inflammatory and comorbid features of patients with severe asthma and frequent exacerbations. Am J Respir Crit Care Med 2017; 195: 302-313.

Lemière C, Boulet L-P, Chaboillez S, et al. Work-exacerbated asthma and occupational asthma: do they really differ? J Allergy Clin Immunol 2013; 131: 704-710.

Dominguez-Ortega J, Barranco P, Rodríguez-Pérez R, et al. Biomarkers in occupational asthma. Curr Allergy Asthma Rep 2016; 16: 63.

Schleich FN, Seidel L, Sele J, et al. Exhaled nitric oxide thresholds associated with a sputum eosinophil coun $\geqslant 3 \%$ in a cohort of unselected patients with asthma. Thorax 2010; 65: 1039-1044.

Belda J, Leigh R, Parameswaran K, et al. Induced sputum cell counts in healthy adults. Am J Respir Crit Care Med 2000; 161: 475-478

Simpson JL, Scott R, Boyle MJ, et al. Inflammatory subtypes in asthma: assessment and identification using induced sputum. Respirology 2006; 11: 54-61.

Schleich FN, Manise M, Sele J, et al. Distribution of sputum cellular phenotype in a large asthma cohort: predicting factors for eosinophilic vs neutrophilic inflammation. BMC Pulm Med 2013; 13: 11.

Moore WC, Hastie AT, Li X, et al. Sputum neutrophil counts are associated with more severe asthma phenotypes using cluster analysis. J Allergy Clin Immunol 2014; 133: 1557-1563.

Wang H, Dasgupta A, Lee K-A, et al. Changing pattern of sputum cell counts during successive exacerbations of chronic obstructive pulmonary disease. COPD 2015; 12: 628-635.

Arron JR, Choy DF, Laviolette M, et al. Disconnect between sputum neutrophils and other measures of airway inflammation in asthma. Eur Respir J 2014; 43: 627-629.

Berry M, Morgan A, Shaw DE, et al. Pathological features and inhaled corticosteroid response of eosinophilic and non-eosinophilic asthma. Thorax 2007; 62: 1043-1049.

Nair P, Aziz-Ur-Rehman A, Radford K. Therapeutic implications of 'neutrophilic asthma'. Curr Opin Pulm Med 2015; 21: 33-38.

Peters MC, McGrath KW, Hawkins GA, et al. Plasma interleukin-6 concentrations, metabolic dysfunction, and asthma severity: a cross-sectional analysis of two cohorts. Lancet Respir Med 2016; 4: 574-584.

Ntontsi P, Loukides S, Bakakos P, et al. Clinical, functional and inflammatory characteristics in patients with paucigranulocytic stable asthma: comparison with different sputum phenotypes. Allergy 2017; 72: 1761-1767.

Gibson PG, Simpson JL, Saltos N. Heterogeneity of airway inflammation in persistent asthma: evidence of neutrophilic inflammation and increased sputum interleukin-8. Chest 2001; 119: 1329-1336.

Jatakanon A, Uasuf C, Maziak W, et al. Neutrophilic inflammation in severe persistent asthma. Am J Respir Crit Care Med 1999; 160: 1532-1539.

Silvestri M, Bontempelli M, Giacomelli M, et al. High serum levels of tumour necrosis factor- $\alpha$ and interleukin- 8 in severe asthma: markers of systemic inflammation? Clin Exp Allergy 2006; 36: 1373-1381.

Casale TB. Biologics and biomarkers for asthma, urticaria, and nasal polyposis. J Allergy Clin Immunol 2017; 139: 1411-1421.

Al-Ramli W, Préfontaine D, Chouiali F, et al. $\mathrm{T}_{\mathrm{H}} 17$-associated cytokines (IL-17A and IL-17F) in severe asthma. J Allergy Clin Immunol 2009; 123: 1185-1187. sputum. J Allergy Clin Immunol 2016; 137: 1433-1446.

Singhania A, Wallington JC, Smith CG, et al. Multitissue transcriptomics delineates the diversity of airway T cell functions in asthma. Am J Respir Cell Mol Biol 2018; 58: 261-270.

Simpson JL, Daly J, Baines KJ, et al. Airway dysbiosis: Haemophilus influenzae and Tropheryma in poorly controlled asthma. Eur Respir J 2016; 47: 792-800.

De Schutter I, Dreesman A, Soetens O, et al. In young children, persistent wheezing is associated with bronchial bacterial infection: a retrospective analysis. BMC Pediatr 2012; 12: 83.

Hynes GM, Hinks TSC. The role of interleukin-17 in asthma: a protective response? ERJ Open Res 2020; 6: 00364-02019.

Chaudhuri R, McSharry C, Brady J, et al. Sputum matrix metalloproteinase-12 in patients with chronic obstructive pulmonary disease and asthma: relationship to disease severity. J Allergy Clin Immunol 2012; 129: 655-663.

Mukhopadhyay S, Sypek J, Tavendale R, et al. Matrix metalloproteinase-12 is a therapeutic target for asthma in children and young adults. J Allergy Clin Immunol 2010; 126: 70-76.

Hunninghake GM, Cho MH, Tesfaigzi Y, et al. MMP12, lung function, and COPD in high-risk populations. N Engl J Med 2009; 361: 2599-2608.

Cooper PR, Mesaros AC, Zhang J, et al. 20-HETE mediates ozone-induced, neutrophil-independent airway hyper-responsiveness in mice. PLoS One 2010; 5: e10235.

Chaudhuri R, Livingston $\mathrm{E}, \mathrm{McMahon} \mathrm{AD}$, et al. Effects of smoking cessation on lung function and airway inflammation in smokers with asthma. Am J Respir Crit Care Med 2006; 174: 127-133. uncontrolled asthma: a randomized controlled trial. J Allergy Clin Immunol 2011; 128: 308-314.

Lazarus SC, Krishnan JA, King TS, et al. Mometasone or tiotropium in mild asthma with a low sputum eosinophil level. N Engl J Med 2019; 380: 2009-2019. 
Cameron EJ, McSharry C, Chaudhuri R, et al. Long-term macrolide treatment of chronic inflammatory airway diseases: risks, benefits and future developments. Clin Exp Allergy 2012; 42: 1302-1312.

Jelić D, Antolović R. From erythromycin to azithromycin and new potential ribosome-binding antimicrobials. Antibiotics 2016; 5: 29.

Schögler A, Kopf BS, Edwards MR, et al. Novel antiviral properties of azithromycin in cystic fibrosis airway epithelial cells. Eur Respir J 2015; 45: 428-439.

Kobayashi Y, Wada H, Rossios C, et al. A novel macrolide/fluoroketolide, solithromycin (CEM-101), reverses corticosteroid insensitivity via phosphoinositide 3-kinase pathway inhibition. Br J Pharmacol 2013; 169: 1024-1034. Culić O, Eraković V, Parnham MJ. Anti-inflammatory effects of macrolide antibiotics. Eur J Pharmacol 2001; 429: 209-229.

Hahn DL. Azithromycin for prevention of exacerbations of COPD. N Engl J Med 2011; 365: 2234-2237.

Amarasiri DL, Pathmeswaran A, de Silva HJ, et al. Response of the airways and autonomic nervous system to acid perfusion of the esophagus in patients with asthma: a laboratory study. BMC Pulm Med 2013; 13: 33 .

Ribó P, Pacheco A, Arrieta P, et al. Gastroesophageal reflux as a cause of chronic cough, severe asthma, and migratory pulmonary infiltrates. Respirol Case Rep 2014; 2: 1-3.

Tomomasa $\mathrm{T}$, Kuroume $\mathrm{T}$, Arai $\mathrm{H}$, et al. Erythromycin induces migrating motor complex in human gastrointestinal tract. Dig Dis Sci 1986; 31: 157-161.

Pennathur A, Tran A, Cioppi M, et al. Erythromycin strengthens the defective lower esophageal sphincter in patients with gastroesophageal reflux disease. Am J Surg 1994; 167: 169-172.

Catnach SM, Fairclough PD. Erythromycin and the gut. Gut 1992; 33: 397-401.

Broad J, Sanger GJ. The antibiotic azithromycin is a motilin receptor agonist in human stomach: comparison with erythromycin. Br J Pharmacol 2013; 168: 1859-1867.

Sifrim D, Matsuo H, Janssens J, et al. Comparison of the effects of midecamycin acetate and azithromycin on gastrointestinal motility in man. Drugs Exp Clin Res 1994; 20: 121-126.

Essilfie A-T, Horvat JC, Kim RY, et al. Macrolide therapy suppresses key features of experimental steroid-sensitive and steroid-insensitive asthma. Thorax 2015; 70: 458-467.

Cameron EJ, Chaudhuri R, Mair F, et al. Randomised controlled trial of azithromycin in smokers with asthma. Eur Respir J 2013; 42: 1412-1415.

Gibson PG, Yang IA, Upham JW, et al. Effect of azithromycin on asthma exacerbations and quality of life in adults with persistent uncontrolled asthma (AMAZES): a randomised, double-blind, placebo-controlled trial. Lancet 2017; 390: 659-668.

Simpson JL, Powell H, Boyle MJ, et al. Clarithromycin targets neutrophilic airway inflammation in refractory asthma. Am J Respir Crit Care Med 2008; 177: 148-155.

Chung KF, Wenzel SE, Brozek JL, et al. Comment on: International ERS/ATS guidelines on definition, evaluation and treatment of severe asthma. Eur Respir J 2014; 44: 267-268.

Ong KY. What's new in the Global Initiative for Asthma 2018 report and beyond. Allergo J Int 2019; 28: 63-72. Laxmanan B, Egressy K, Murgu SD, et al. Advances in bronchial thermoplasty. Chest 2016; 150: 694-704.

Schuhmann M, Konietzke P, Wielpütz M, et al. Reduction of bronchial wall thickness and hyperinflation on quantitative CT after bronchial thermoplasty for severe asthma. Eur Respir J 2017; 50: PA3028.

Thomson NC. Novel approaches to the management of noneosinophilic asthma. Ther Adv Respir Dis 2016; 10: 211-234.

Ulrik CS. Asthma and obesity: is weight reduction the key to achieve asthma control? Curr Opin Pulm Med 2016 22: 69-73.

Adeniyi FB, Young T. Weight loss interventions for chronic asthma. Cochrane Database Syst Rev 2012; 7 : CD009339. meta-analysis. J Allergy Clin Immunol Pract 2018; 6: 570-581.

Zhu L, Ciaccio CE, Casale TB. Potential new targets for drug development in severe asthma. World Allergy Organ J 2018; 11: 30. sputum neutrophils: a randomized, placebo-controlled clinical trial. Clin Exp Allergy 2012; 42: 1097-1103.

O'Byrne PM, Metev H, Puu M, et al. Efficacy and safety of a CXCR2 antagonist, AZD5069, in patients with uncontrolled persistent asthma: a randomised, double-blind, placebo-controlled trial. Lancet Respir Med 2016; 4: 797-806.

Leaker BR, Barnes PI, O'Connor B. Inhibition of LPS-induced airway neutrophilic inflammation in healthy volunteers with an oral CXCR2 antagonist. Respir Res 2013; 14: 137.

Busse WW, Holgate S, Kerwin E, et al. Randomized, double-blind, placebo-controlled study of brodalumab, a human anti-IL-17 receptor monoclonal antibody, in moderate to severe asthma. Am J Respir Crit Care Med 2013; 188: 1294-1302.

5 Verona Pharma. Verona Pharma reports Positive Results From RPL554 Dose-Finding Study. www. veronapharma.com/media/verona-pharma-reports-positive-results-rpl554-dose-finding-study/ Date last updated: 15 March 2016.

Singh D, Abbott-Banner K, Bengtsson $\mathrm{T}$, et al. The short-term bronchodilator effects of the dual phosphodiesterase 3 and 4 inhibitor RPL554 in COPD. Eur Respir J 2018; 52: 1801074.

Wenzel SE, Barnes PJ, Bleecker ER, et al. A randomized, double-blind, placebo-controlled study of tumor necrosis factor- $\alpha$ blockade in severe persistent asthma. Am J Respir Crit Care Med 2009; 179: 549-558.

Antoniu SA. Cytokine antagonists for the treatment of asthma: progress to date. BioDrugs 2009; 23: 241-251. Hernandez ML, Mills K, Almond M, et al. IL-1 receptor antagonist red
inflammation in healthy volunteers. J Allergy Clin Immunol 2015; 135: 379-385.

Marks-Konczalik J, Costa M, Robertson J, et al. A post-hoc subgroup analysis of data from a six month clinical trial comparing the efficacy and safety of losmapimod in moderate-severe COPD patients with $\leqslant 2 \%$ and $>2 \%$ blood eosinophils. Respir Med 2015; 109: 860-869.

Patel NR, Cunoosamy DM, Fagerås M, et al. The development of AZD7624 for prevention of exacerbations in COPD: a randomized controlled trial. Int J Chron Obstruct Pulmon Dis 2018; 13: 1009-1019. 
Humbert M, de Blay F, Garcia G, et al. Masitinib, a c-kit/PDGF receptor tyrosine kinase inhibitor, improves disease control in severe corticosteroid-dependent asthmatics. Allergy 2009; 64: 1194-1201.

Khindri S, Cahn A, Begg M, et al. A multicentre, randomized, double-blind, placebo-controlled, crossover study to investigate the efficacy, safety, tolerability, and pharmacokinetics of repeat doses of inhaled nemiralisib in adults with persistent, uncontrolled asthma. J Pharmacol Exp Ther 2018; 367: 405-413.

Winkler DG, Faia KL, DiNitto JP, et al. PI3K- $\delta$ and PI3K- $\gamma$ inhibition by IPI-145 abrogates immune responses and suppresses activity in autoimmune and inflammatory disease models. Chem Biol 2013; 20: 1364-1374.

Doukas J, Eide L, Stebbins K, et al. Aerosolized phosphoinositide 3-kinase $\gamma / \delta$ inhibitor TG100-115 [3-[2,4-diamino-6-(3-hydroxyphenyl)pteridin-7-yl]phenol] as a therapeutic candidate for asthma and chronic obstructive pulmonary disease. J Pharmacol Exp Ther 2009; 328: 758-765.

Koziol-White CJ, Zhang J, Yoo E, et al. A narrow spectrum kinase inhibitor, RV1729, induces bronchodilation of human small airways and rescues agonist-induced desensitization of the 2 adrenoreceptor (2AR). Am J Respir Crit Care Med 2016; 193: A6841.

Mukaida N. Pathophysiological roles of interleukin-8/CXCL8 in pulmonary diseases. Am J Physiol Lung Cell Mol Physiol 2003; 284: L566-L577.

Xu X, Jackson PL, Tanner S, et al. A self-propagating matrix metalloprotease-9 (MMP-9) dependent cycle of chronic neutrophilic inflammation. PLoS One 2011; 6: e15781.

Traves SL, Culpitt SV, Russell REK, et al. Increased levels of the chemokines GRO $\alpha$ and MCP-1 in sputum samples from patients with COPD. Thorax 2002; 57: 590-595.

Sergejeva S, Ivanov S, Lötvall J, et al. Interleukin-17 as a recruitment and survival factor for airway macrophages in allergic airway inflammation. Am J Respir Cell Mol Biol 2005; 33: 248-253.

Fogli LK, Sundrud MS, Goel S, et al. T cell-derived IL-17 mediates epithelial changes in the airway and drives pulmonary neutrophilia. J Immunol 2013; 191: 3100-3111.

Ricciardolo FLM, Sorbello V, Vallese D, et al. Expression of nasal and bronchial IL-17F and related cytokines in frequent exacerbators with neutrophilic severe asthma. Eur Respir J 2016; 48: PA4176.

Chen R, Zhang Q, Chen S, et al. IL-17F, rather than IL-17A, underlies airway inflammation in a steroid-insensitive toluene diisocyanate-induced asthma model. Eur Respir J 2019; 53: 1801510. chronic ozone-induced airway inflammation in mice. Biomed Pharmacother 2016; 83: 247-256. Controlled Severe Asthma. https://clinicaltrials.gov/ct2/show/NCT03299686/ Date last updated: 12 August 2020.

Miller M. Phosphodiesterase inhibition in the treatment of autoimmune and inflammatory diseases: current status and potential. J Recept Ligand Channel Res 2015; 8: 19-30.

Lucci G, Mariotti F, Santoro D, et al. Safety, tolerability and pharmacokinetics of CHF 6001, a novel selective inhaled PDE4 inhibitor, in healthy volunteers. Eur Respir J 2016; 48: PA4086.

Erin EM, Leaker BR, Nicholson GC, et al. The effects of a monoclonal antibody directed against tumor necrosis factor- $\alpha$ in asthma. Am J Respir Crit Care Med 2006; 174: 753-762.

He Y, Peng S, Xiong W, et al. Association between polymorphism of interleukin-1 beta and interleukin-1 receptor antagonist gene and asthma risk: a meta-analysis. ScientificWorldJournal 2015; 2015: 685684.

Konno S, Gonokami Y, Kurokawa M, et al. Cytokine concentrations in sputum of asthmatic patients. Int Arch Allergy Immunol 1996; 109: 73-78.

(azzarella G, Grella E, D'Auria D, et al. Phenotypic features of alveolar monocytes/macrophages and IL-8 gene activation by IL-1 and TNF-alpha in asthmatic patients. Allergy 2000; 55: Suppl. 61, 36-41.

Sousa AR, Lane SJ, Nakhosteen JA, et al. Expression of interleukin-1 beta (IL-1beta) and interleukin-1 recepto antagonist (IL-1ra) on asthmatic bronchial epithelium. Am J Respir Crit Care Med 1996; 154: 1061-1066.

Hernandez ML, Wagner JG, Kala A, et al. Vitamin E, $\gamma$-tocopherol, reduces airway neutrophil recruitment after inhaled endotoxin challenge in rats and in healthy volunteers. Free Radic Biol Med 2013; 60: 56-62. in severe, steroid-resistant asthma. Am J Respir Crit Care Med 2017; 196: 283-297.

Church LD, McDermott MF. Canakinumab: a human anti-IL-1 $\beta$ monoclonal antibody for the treatment of cryopyrin-associated periodic syndromes. Expert Rev Clin Immunol 2010; 6: 831-841.

Chu DK, Al-Garawi A, Llop-Guevara A, et al. Therapeutic potential of anti-IL-6 therapies for granulocytic airway inflammation in asthma. Allergy Asthma Clin Immunol 2015; 11: 14.

Banno A, Reddy AT, Lakshmi SP, et al. PPARs: key regulators of airway inflammation and potential therapeutic targets in asthma. Nucl Receptor Res 2018; 5: 101306.

GlaxoSmithKline. A Phase 2a Study to Evaluate the Effects of Sirukumab in Subjects with Severe Poorly Controlled Asthma. https://clinicaltrials.gov/ct2/show/NCT02794519/ Date last updated: 31 August 2018.

9 Lea S, Harbron $\mathrm{C}$, Khan $\mathrm{N}$, et al. Corticosteroid insensitive alveolar macrophages from asthma patients; synergistic interaction with a p38 mitogen-activated protein kinase (MAPK) inhibitor. Br J Clin Pharmacol 2015; 79: 756-766.

Pfizer. An Evaluation of PF-03715455 in Moderate to Severe Uncontrolled Asthma. https://clinicaltrials.gov/ct2/ show/NCT02219048/ Date last updated: 12 June 2019.

Reber L, Da Silva CA, Frossard N. Stem cell factor and its receptor c-Kit as targets for inflammatory diseases. Eur J Pharmacol 2006; 533: 327-340.

Southworth T, Plumb J, Gupta V, et al. Anti-inflammatory potential of PI3K $\delta$ and JAK inhibitors in asthma patients. Respir Res 2016; 17: 124.

Zhang L-B, He M. Effect of mesenchymal stromal (stem) cell (MSC) transplantation in asthmatic animal models: a systematic review and meta-analysis. Pulm Pharmacol Ther 2019; 54: 39-52.

Yoo EJ, Ojiaku CA, Sunder K, et al. Phosphoinositide 3-kinase in asthma: novel roles and therapeutic approaches. Am J Respir Cell Mol Biol 2017; 56: 700-707. 